\title{
A new multiwavelength lepto-hadronic model of astrophysical jet emission
}

\author{
Samia Drappeau*t \\ IRAP; Université de Toulouse; UPS-OMP; CNRS; \\ 9 Av. colonel Roche, BP 44346, F-31028 Toulouse cedex 4, France \\ E-mail: samia.drappeaulirap.omp.eu

\section{Sera Markoff} \\ Astronomical Institute "Anton Pannekoek", University of Amsterdam, \\ Postbus 94249, 1090 GE Amsterdam, The Netherlands
}

\section{Stéphane Corbel}

Laboratoire AIM (CEA/IRFU - CNRS/INSU - Université Paris Diderot), CEA DSM/IRFU/SAp, F-91191 Gif-sur-Yvette, France

\section{Michael Nowak}

Massachusetts Institute of Technology, Kavli Institute for Astrophysics,

Cambridge, MA 02139, USA

\section{Jörn Wilms}

Dr. Remeis-Sternwarte Bamberg, Astronomisches Institut der Universität Erlangen-Nürnberg,

ECAP, D-96049 Bamberg, Germany

\begin{abstract}
In the last few years, the rapid development of gamma-ray observations has open a new window on X-ray binaries (XRBs) sources. In this talk we introduce a new lepto-hadronic model that is based on successful work fitting the lower energy, broadband spectra of XRBs in the compact jetdominated state. Protons (and electrons) are accelerated throughout the jet and cool via radiation and inelastic collisions, and we calculate spectral energy distributions (SEDs) including both hadronic and leptonic induced processes. This model can also be scaled to describe the SEDs of low-luminosity active galactic nuclei such as our own Galactic Center black hole Sgr A*.
\end{abstract}

Frontiers of Fundamental Physics 14

15-18 July 2014

Aix Marseille University (AMU) Saint-Charles Campus, Marseille, France

\footnotetext{
${ }^{*}$ Speaker.

${ }^{\dagger}$ This work was supported by a Netherlands Organisation for Scientific Research (NWO) Vidi Fellowship.
} 


\section{Introduction}

The content of jets of accreting black hole systems remains an open question. Successful analyses of multiwavelength observations, from radio to X-rays, of different sources with only leptonic radiative processes have made the leptonic jet model the most favoured jet emission model. However, analyses of the cosmic ray spectrum show the proton as its dominant component, which means that somewhere in the Universe exist sources capable of accelerating hadrons to very high energy. Jets from accreting black holes are considered as one of the possible candidates of such acceleration sites. That and the recent detection of baryons in 4U1630-47 [8] encourage us to revise the traditional leptonic model of emission and to consider the contribution of hadronic processes to the overall radiation.

A large volume of published studies examine the contribution of hadronic processes in AGN jet emission (e.g. [6, 3, 23, 34, 20, 29, 4]). Over the past few years, several groups (e.g. [36, 5, 30, 37]) have adapted these hadronic models of AGN jets to XRB jets. These studies all share the common approach of modelling only the non-thermal emission from the jet. However, the fundamental plane of black hole accretion $[28,12,33]$ as well as works based on general relativistic magnetohydrodynamical (GRMHD) simulations [26, 27, 2, 9] support the idea that accretion discs, bases of jets and jets themselves are all connected, forming one inflow-outflow system. To understand the power in jets and their content, and to model multiwavelength observations of accreting black holes, it is therefore essential to study the system as a whole, thermal and non-thermal sources of emission from the accretion disc and the jets altogether.

The aim of this talk is to present a new spectral model which calculates continuum emission from lepto-hadronic processes occurring in accreting black hole sources from thermal and nonthermal particles distributions. Our work is based on a leptonic jet model which has been successful in fitting the lower energy, broadband spectra of XRBs in the compact jet-dominated state as well as spectra of low-luminosity (LL) and Fanaroff-Riley Type 1 (FR I) AGN [25, 24, 21, 22].

\section{Spectral model of jets}

The lepto-hadronic spectral jet model used in this work is based on a leptonic jet model developed by [25] (see also [11, 10, 13]). Accelerated protons interacting with their surroundings naturally produce photons with energies in the gamma-ray range. With the development of gamma-ray observatories, we have the opportunity to test the nature of accreting black hole jets by comparing the resulting emission from leptonic versus lepto-hadronic models. And so, we modify the original leptonic model to incorporate additional physics. These modifications allow us to investigate the contribution of each population of particles in the jet to the overall emission in general, and to the gamma-ray band in particular.

The details of the physics and the description of the main parameters of the original leptonic model are presented in the appendix of [25]. Only a brief summary is given here, with a focus on the modifications made to incorporate the treatment of hadronic emission.

In our model, the jets are launched perpendicularly to the plane of the accretion disc, as shown in Figure 1. In cylindrical coordinates, the z-axis is taken as the symmetry axis of the jet and $\theta$ is the angle this axis makes with the line of sight. A nozzle of constant radius $r_{0}$ and height $h_{0}$ acts as 


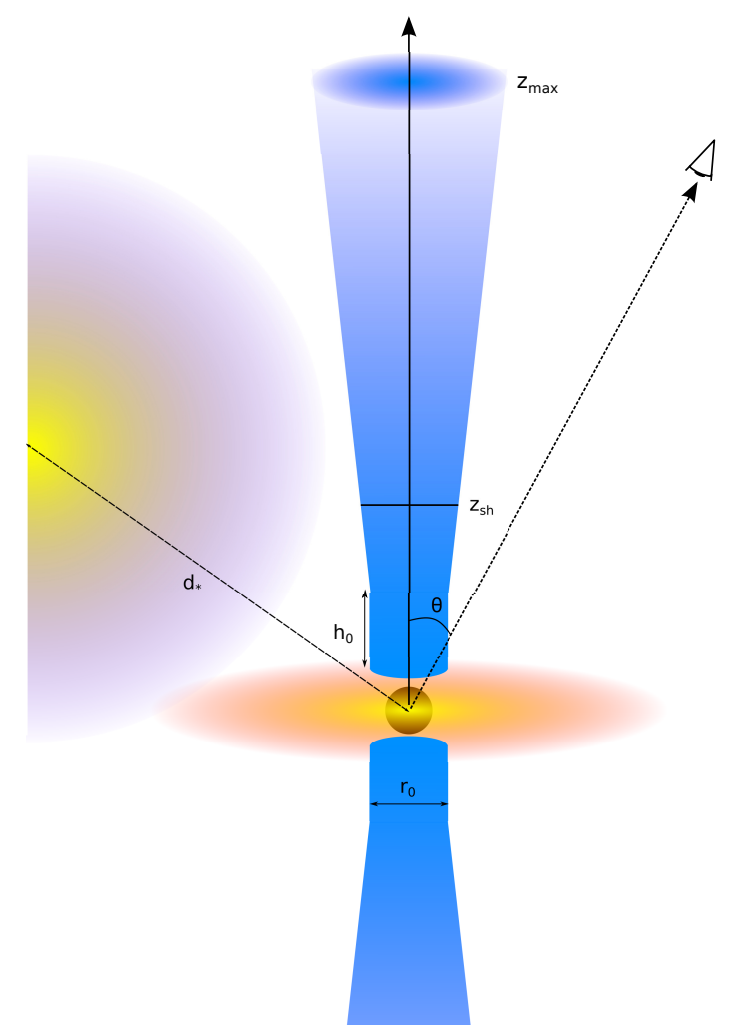

Figure 1: Sketch presenting the different elements and relevant geometrical parameters of the jet model. See text for definition of the parameters.

the base of the jet. Beyond, the jet expands sideways adiabatically, until a maximum height $z_{\max }$. The model does not explicitly treat particle acceleration. Instead, it assumes a location $z_{\mathrm{sh}}$ along the jet beyond which a significant fraction of the leptons and hadrons are accelerated to a power-law energy distribution. The radius $r_{0}$ and the height $h_{0}$ of the nozzle, and the location $z_{\text {sh }}$ are some of the seven main parameters determining the properties of the jet. The others are the input jet power $N_{\mathrm{j}}$, the temperature $T_{\mathrm{e}}$ of the relativistic thermal electrons entering the base of the jet and the equipartition factor $k$, representing the ratio between magnetic and particle energy densities. The ion-to-electron temperature ratio $T_{\mathrm{i}} / T_{\mathrm{e}}$ completes this list.

\section{Hadronic emission}

As already noted, the model does not explicitly treat particle acceleration. It assumes that beyond a location $z_{\mathrm{sh}}$ in the jet, a fraction of the thermal Maxwell-Boltzmann distribution of particles is accelerated to a power-law distribution. Regarding the protons, we follow the findings of [7] and inject a power-law distribution in momentum instead of energy. These accelerated protons can reach a maximum momentum given by the balance of acceleration and cooling losses. By equating the acceleration rate and the cooling rate, given by [3], we express the maximum momentum $p c_{\max }$ a proton can get accelerated to in the jet. Accelerated protons in jets mainly cool via inelastic collisions with matter and radiation. Synchrotron and Compton cooling can also be considered, 
although, in the present version of the code, these interactions have not been implemented yet for the protons. Protons have another constraint on the maximum momentum they can attain which is given by the Hillas criterion [14]. The criterion stipulates that only protons with gyroradius $r_{\mathrm{gy}}$ not exceeding the size of the acceleration region remain confined in the jet. Therefore, the maximum momentum attained by the accelerated protons in the jet is the minimum between the momentum given by equating the acceleration and cooling rate and by the Hillas criterion.

Accelerated protons interacting via inelastic collisions with matter and radiation fields, create secondary particles. Several groups have worked on expressing the resulting spectrum of these secondary particles. [18] provide useful parametrisation of energy spectra of all secondary particles produced in proton-proton interactions. Their results were obtained by fitting the spectra of secondary particles produced in proton-proton inelastic collisions simulated by the SIBYLL code. However, their simple analytical approximation is only valid for energies above $100 \mathrm{GeV}$. [15] (and its extension [16]) present parametrised formulae allowing the calculation of cross-sections as well as spectra of stable secondary particles of proton-proton interactions for proton energies from the pion production threshold up to $10^{5} \mathrm{GeV}$. Again, up-to-date Monte-Carlo simulations have been used to derive these parametrisations. The work of [15] improves the parametrisation of the crosssection near the pion production threshold by incorporating two baryon resonance excitations: the $\Delta(1232)$ resonance and the resonances around $1600 \mathrm{MeV} / c^{2}$.

Because it models the cross-section more accurately near the pion production threshold, we chose to use the parametrisations of [15], and its extension [16], to model the proton-proton interactions occurring in our jets. Finally, to express the energy distribution of photons, electrons and neutrinos produced in interactions of relativistic protons with a radiation field, we use the simple analytical parametrisations provided by [17].

One major modification done to the leptonic version of the jet model consists of determining the steady-state energy distributions of the secondary electrons and positrons to calculate their synchrotron and inverse-Compton scattering radiation. These distributions are assumed to be isotropic. All the calculations of energy distributions and luminosities are done in the jet frame, except in the case of proton-proton interactions. In this case, it is important to derive the proton distribution in the jet as seen by the observer, when we calculate secondary particle distributions. This is due to the fact that the parametrisations of the interaction cross-section and decay functions are expressed in the observer frame.

While the accelerated protons can interact with radiation fields naturally provided by leptonic interactions, stellar or disc emission, accreting black holes need an external source of thermal protons to give rise to $\gamma$-ray emission from proton-proton interactions. High-mass X-ray binaries are ideal candidates for this situation. The stellar wind of their massive companion star can be strong enough to reach and interact with the jets. The thermal protons of the wind interact with the relativistic protons in the jets, producing $\gamma$-ray photons with energy above the $\mathrm{GeV}$ range. If the wind is clumpy, the rate of proton-proton interactions may increase temporarily, resulting in $\gamma$-ray flares (see e.g., [31, 1, 35, 32]). However, our work focuses on the steady-state emission of an accreting black hole in a weakly accreting state, we thus choose to model the stellar wind of the companion star by a stationary spherically symmetric wind [19].

To summarise, based on useful parametrisations by [16] and [17], we have developed routines that calculate the spectra of photon, electron/positron and neutrino secondary particles from 


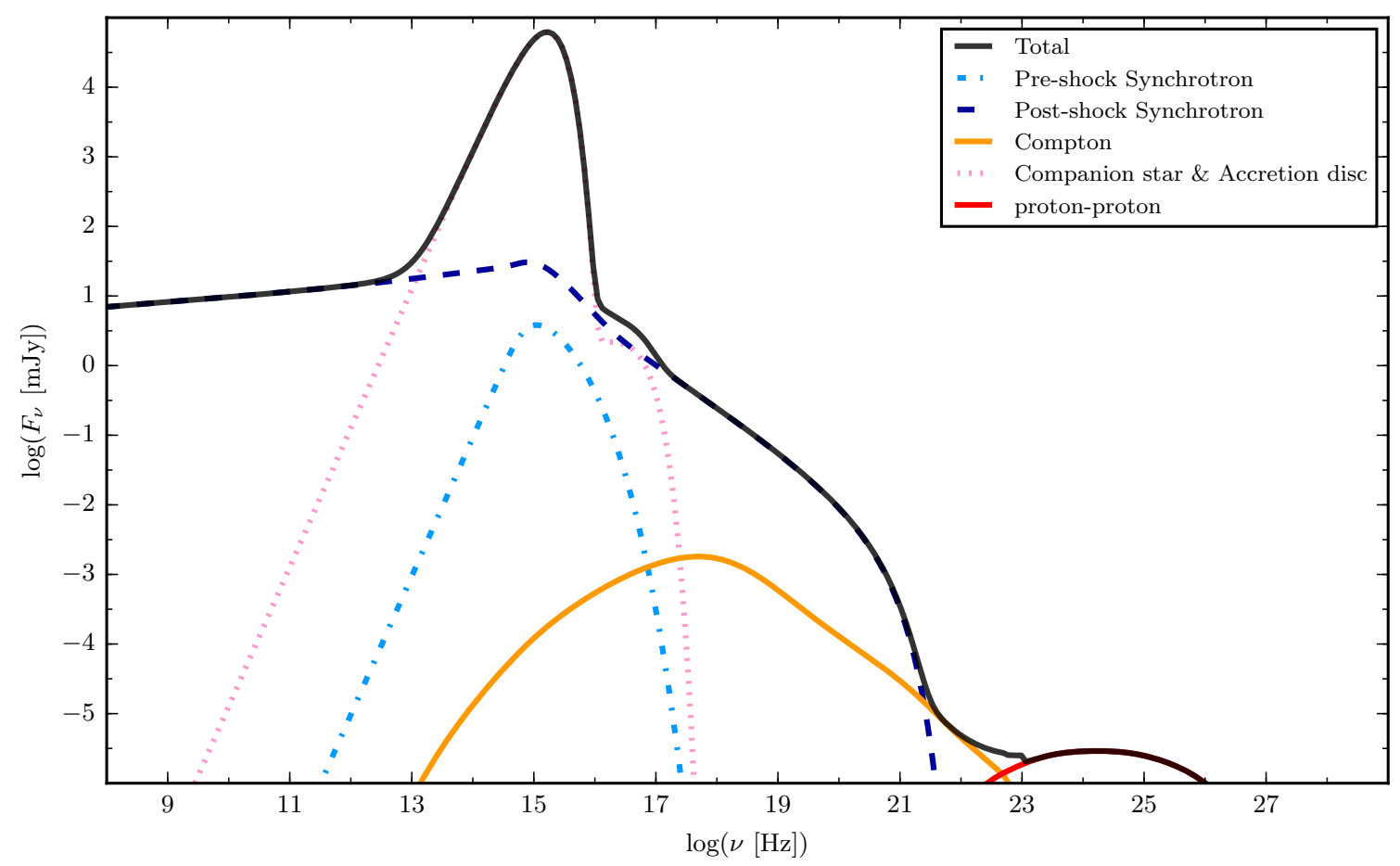

Figure 2: SED representing the different components of the model contributing to the total emission: synchrotron emission from primary electrons with thermal and non-thermal distributions (resp. light blue dashed-dotted and dark blue dashed lines), inverse-Compton emission (orange solid line), black-body emission from the accretion disc and a companion star (pink dotted line) and the $\gamma$ emission from proton-proton interaction (red solid line). The black solid line represents the total emission.

proton-proton and proton- $\gamma$ interactions. Then, we have implemented these routines in a modified version of the original leptonic model $[25,21]$, where the protons are now accelerated alongside the electrons in the jets. We have also added a simple stellar wind model to account for cases where the companion star produces a powerful wind which interacts with the jets. Finally, the new jet model produces steady-state spectral energy distributions which include both hadronic and leptonic induced processes from secondary electrons and positrons as well as primary particles. Fig. 2 presents an example of SED procuded by the model.

\section{Conclusion}

This talk presents a new spectral model which calculates the continuum emission from thermal and non-thermal lepto-hadronic processes occurring in jets from accreting black holes. Our work consisted of modifying a successful leptonic jet model, to account for proton acceleration along the jet and its resulting emission. One of the modifications done to the leptonic jet model was to calculate the steady-state energy distribution of the protons along the jets. The distribution of primary protons was then fed to hadronic routines we developed and implemented in the model. These routines calculate the energy distributions of the photons, electrons, positrons, and neutrinos from 
proton-proton and proton-photon interactions. Their kernels are based on two useful parametrisations of the spectra of secondary particles from hadronic interactions available in the literature. Finally, the steady-state energy distributions of the secondary electrons and positrons are derived to calculate their contribution to the overall spectrum. Our goal is to use this new spectral model to analyse broad-band spectra from radio to $\gamma$-rays of X-ray binary as well as LLAGN and FR I sources.

By providing the community with predictions for the electromagnetic emission as well as neutrino signals of accreting black holes, this work allows us to perform more rigourous multiwavelength analyses and opens the door to pioneer multi-messenger analyses of these objects.

\section{References}

[1] A. T. Araudo, V. Bosch-Ramon, and G. E. Romero, High-energy emission from jet-clump interactions in microquasars, $A \& A, 503$ (2009), 673

[2] K. Beckwith, J. F. Hawley, and J. H. Krolik, The Influence of Magnetic Field Geometry on the Evolution of Black Hole Accretion Flows: Similar Disks, Drastically Different Jets, ApJ 678 (2008), 1180

[3] M. C. Begelman, B. Rudak, and M. Sikora, Consequences of relativistic proton injection in active galactic nuclei, ApJ, 362 (1990), 38

[4] V. Bosch-Ramon, Theoretical overview on high-energy emission in microquasars, Ap\&SS, 309 (2007), 321

[5] V. Bosch-Ramon, F. A. Aharonian, and J. M. Paredes, Electromagnetic radiation initiated by hadronic jets from microquasars in the ISM, A\&A, 432 (2005), 609

[6] C. D. Dermer, Secondary production of neutral pi-mesons and the diffuse galactic gamma radiation, A\&A, 157 (1986), 223

[7] C. D. Dermer, Diffuse Galactic Gamma Rays from Shock-Accelerated Cosmic Rays, Physical Review Letters, 109 (2012)

[8] M. Díaz Trigo, J. C. A. Miller-Jones, S. Migliari, J. W. Broderick, and T. Tzioumis, Baryons in the relativistic jets of the stellar-mass black-hole candidate 4U1630-47, Nature, 504 (2013), 260

[9] S. Dibi, S. Drappeau, P. C. Fragile, S. Markoff, and J. Dexter, General relativistic magnetohydrodynamic simulations of accretion on to Sgr A*: how important are radiative losses?, MNRAS, 426 (2012), 1928

[10] H. Falcke, The Nuclear Jet in M81, ApJL, 464:L67, June 1996.

[11] H. Falcke and P. L. Biermann, The jet-disk symbiosis. I. Radio to X-ray emission models for quasars. $A \& A, 293$ (1995), 665

[12] H. Falcke, E. Körding, and S. Markoff, A scheme to unify low-power accreting black holes. Jet-dominated accretion flows and the radio/X-ray correlation, $A \& A, 414$ (2004), 895

[13] H. Falcke and S. Markoff, The jet model for Sgr A*: Radio and X-ray spectrum, A\&A, 362 (2000), 113

[14] A. M. Hillas, The Origin of Ultra-High-Energy Cosmic Rays, ARAA, 22 (1984), 425

[15] T. Kamae, N. Karlsson, T. Mizuno, T. Abe, and T. Koi, Parameterization of $\gamma, e^{+/-}$, and Neutrino Spectra Produced by p-p Interaction in Astronomical Environments, ApJ, 647 (2006), 692 
[16] N. Karlsson and T. Kamae, Parameterization of the Angular Distribution of Gamma Rays Produced by p-p Interaction in Astronomical Environments, ApJ, 674 (2008), 278

[17] S. R. Kelner and F. A. Aharonian, Energy spectra of gamma rays, electrons, and neutrinos produced at interactions of relativistic protons with low energy radiation, $P h R v D, 78$ (2008)

[18] S. R. Kelner, F. A. Aharonian, and V. V. Bugayov, Energy spectra of gamma rays, electrons, and neutrinos produced at proton-proton interactions in the very high energy regime, PhRvD, 74 (2006)

[19] H. J. G. L. M. Lamers and J. P. Cassinelli, Introduction to Stellar Winds, Cambridge University Press, Cambridge, UK, 1999.

[20] R. Mahadevan, R. Narayan, and J. Krolik, Gamma-Ray Emission from Advection-dominated Accretion Flows around Black Holes: Application to the Galactic Center, ApJ, 486 (1997), 268

[21] D. Maitra, S. Markoff, C. Brocksopp, M. Noble, M. Nowak, and J. Wilms, Constraining jet/disc geometry and radiative processes in stellar black holes XTE J1118+480 and GX 339-4, MNRAS, 398 (2009), 1638

[22] D. Maitra, J. M. Miller, S. Markoff, and A. King, A Jet Model for the Broadband Spectrum Of the Seyfert 1 Galaxy NGC 4051, ApJ, 735 (2011), 107

[23] K. Mannheim, The proton blazar, A\&A, 269 (1993), 67

[24] S. Markoff, M. Nowak, A. Young, H. L. Marshall, C. R. Canizares, A. Peck, M. Krips, G. Petitpas, R. Schödel, G. C. Bower, P. Chandra, A. Ray, M. Muno, S. Gallagher, S. Hornstein, and C. C. Cheung, Results from an Extensive Simultaneous Broadband Campaign on the Underluminous Active Nucleus M81*: Further Evidence for Mass-scaling Accretion in Black Holes, ApJ, 681 (2008), 905

[25] S. Markoff, M. A. Nowak, and J. Wilms, Going with the Flow: Can the Base of Jets Subsume the Role of Compact Accretion Disk Coronae?, ApJ, 635 (2005), 1203

[26] J. C. McKinney, General relativistic magnetohydrodynamic simulations of the jet formation and large-scale propagation from black hole accretion systems, MNRAS, 368 (2006), 1561

[27] J. C. McKinney and R. Narayan, Disc-jet coupling in black hole accretion systems - I. General relativistic magnetohydrodynamical models, MNRAS, 375 (2007), 513

[28] A. Merloni, S. Heinz, and T. di Matteo, A Fundamental Plane of black hole activity, MNRAS, 345 (2003), 1057

[29] A. Mucke, J. P. Rachen, R. Engel, R. J. Protheroe, and T. Stanev, Photohadronic processes in astrophysical environments, PASA, 16:160-6, August 1999.

[30] M. Orellana, P. Bordas, V. Bosch-Ramon, G. E. Romero, and J. M. Paredes, Leptonic secondary emission in a hadronic microquasar model, A\&A, 476 (2007), 9

[31] S. P. Owocki, G. E. Romero, R. H. D. Townsend, and A. T. Araudo, Gamma-Ray Variability from Wind Clumping in High-Mass X-Ray Binaries with Jets, ApJ, 696 (2009), 690

[32] M. Perucho and V. Bosch-Ramon, 3D simulations of microquasar jets in clumpy stellar winds, A\&A, 539 (2012), A57

[33] R. M. Plotkin, S. Markoff, B. C. Kelly, E. Körding, and S. F. Anderson, Using the Fundamental Plane of black hole activity to distinguish X-ray processes from weakly accreting black holes, MNRAS, 419 (2012), 267

[34] J. P. Rachen and P. L. Biermann, Extragalactic Ultra-High Energy Cosmic-Rays - Part One Contribution from Hot Spots in Fr-II Radio Galaxies, A\&A, 272 (1993), 161 
[35] G. E. Romero, M. V. Del Valle, and M. Orellana, Gamma-ray absorption and the origin of the gamma-ray flare in Cygnus X-1, A\&A, 518 (2010), A12

[36] G. E. Romero, D. F. Torres, M. M. Kaufman Bernadó, and I. F. Mirabel, Hadronic gamma-ray emission from windy microquasars, $A \& A, 410$ (2003), L1

[37] G. E. Romero and G. S. Vila, The proton low-mass microquasar: high-energy emission, A\&A, 485 (2008), 623 